\title{
Eficacia y eficiencia del tratamiento antituberculoso en jurisdicciones sanitarias de Morelos
}

\author{
Ma. de Lourdes García-García, M.C., Dr. en C.,1) Ma. Eugenia Mayar-Maya, Lic. en Inf.,(1) \\ Leticia Ferreyra-Reyes, M.C., ${ }^{(1)}$ Manuel Palacios-Martínez, M.C., ${ }^{(1)}$ Carlos Alvarez-García,Téc. en Inf., ${ }^{(1)}$ \\ José Luis Valdespino-Gómez, M.C., M.S.P. ${ }^{(1)}$
}

\begin{abstract}
García-García ML, Mayar-Maya ME, Ferreyra-Reyes L, Palacios-Martínez M, Alvarez-García C, Valdespino-Gómez JL. Eficacia y eficiencia del tratamiento antituberculoso en jurisdicciones sanitarias de Morelos. Salud Publica Mex 1998;40:421-429.
\end{abstract}

\section{Resumen}

Objetivo. Evaluar la eficacia y la eficiencia del tratamiento antituberculoso administrado por la Secretaría de Salud (SSA) en las jur isdicciones sanitarias de C uernavaca y C uautla, estado de Morelos, en el periodo 1992-1996. Material y métodos. Se hizo una revisión retrospectiva de las tarjetas de control de tratamiento en pacientes tuberculosos. Se utilizaron las definiciones contenidas en la N orma 0 ficial para el Control y Prevención de la Tuberculosis en la Atención Primaria a la Salud. La información fue vaciada en formatos estandarizados y se analizó mediante el programa SAS y Epi Info. Se visitaron las 149 unidades de atención primaria y los cuatro hospitales de la zona de estudio. Resultados. Se encontraron las tarjetas correspondientes a 288 pacientes, de los cuales 260 eran de casos nuevos. Estos pacientes recibieron en conjunto 311 tratamientos, de los cuales $85 \%$ fueron supervisados. Las tarjetas revisadas correspondieron al $60 \%$ de los casos notificados por la SSA en el mismo periodo. El grupo de pacientes tuberculosos tuvo mayor edad que la media poblacional y mayor probabilidad de pertenecer a los estratos socioeconómicos medio y bajo que la población general del área. Se analizaron 246 esquemas de tratamiento y se encontró que $32 \%$ presentó curación bacteriológica; $26 \%$, curación probable; $18 \%$ abandonó el tratamiento; $1 \%$ fracasó en el tratamiento, y $3 \%$ murió durante el tratamiento. En $20 \%$ de los casos se desconoció el resultado del tratamiento. La frecuencia de
García-García ML, Mayar-Maya ME, Ferreyra-Reyes L, Palacios-Martínez M, Alvarez-García C, Valdespino-Gómez JL. Efficacy and efficiency of anti-tuberculosis treatment in 2 sanitary jurisdictions of Morelos, Mexico. Salud Publica Mex 1998;40:421-429.

\section{A bstract}

Objective. To evaluate the efficacy and efficiency of tuberculosis treatment administered by the Secretaría de Salud (SSA) in the sanitary jurisdictions of C uernavaca and Cuautla, Morelos, for the 1992-1996 period by retrospectively reviewing tuberculosis treatment control cards. Material and methods. 0 fficial Norm for Tuberculosis Prevention and Control in Primary Care Units outcome definitions were used. Data was collected on standardized forms and analyzed with SAS and Epi Info programs; 149 primary care units and 4 hospitals in the study area were visited. Results. There were found 288 patient cards, of which 260 were new cases. These patients received 311 treatments of which $85 \%$ were directly observed. Reviewed cards represented $60 \%$ of SSA notified cases for this period. There were analyzed 246 treatments of which $32 \%$ were bacteriological cures, $26 \%$ probable cures, $18 \%$ dropouts, $1 \%$ failures and $3 \%$ deaths. In $20 \%$ of treatments the outcome was unknown. Cure rate was better in new cases $(61 \%)$ than in retreatments $(38 \%), p<0.01$. Efficacy of treatment was $71 \%$ and efficiency $58 \%$. Patients receiving retreatment abandoned it more frequently (32\%) than new cases $(16 \%), p<0.01$. A statistically significant association was found between abandoned treatment and being retreated $(O \mathrm{R}=3.3, \mathrm{Cl} 95 \%$ $1.3-8.5, p=0.01$ ) or belonging to a lower socioeconomic level $(O R=2.3, C \mid 95 \% 1.0-4.9, p=0.04)$. In the 34 retreatment programs, 22 were initiated after abandonment, fail-

(1) Secretaría A cadémica. Instituto N acional de Salud Pública, México.

Fecha de recibido: 24 de febrero de 1997 - Fecha de aprobado: 22 de abril de 1998

Solicitud de sobretiros: Dra. Ma. de Lourdes García. Subdirección de Gestión de Proyectos, Secretaría A cadémica, Instituto Nacional de Salud Pública. Av. Universidad N 0. 655, colonia Santa María A huacatitlán. 62508 Cuernavaca, Morelos, México. Correo electrónico: garcigar. insp3. insp.mx 
curación fue mayor en los casos nuevos (61\%) que en los retratamientos $(38 \%)(p<0.01)$. Los pacientes en retratamiento lo abandonaron con mayor frecuencia (32\%) que los casos nuevos $(16 \%)(p<0.01)$. La eficacia del tratamiento fue de $71 \%$, y la eficiencia, de $58 \%$. Se encontró asociación estadísticamente significativa entre abandono de tratamiento y retratamiento (RM 3.3, IC 95\% 1.3-8.5, $p=0.01$ ) 0 nivel socioeconómico bajo (RM 2.3, IC 95\% 1.0-4.9, $p=$ $0.04)$. En los 34 esquemas de retratamiento, 11 lo iniciaron por abandono del tratamiento previo, y 11 por fracaso 0 recaída. Conclusiones. La proporción de pacientes que se curan es de solamente $58 \%$, lo cual está por debajo del mínimo recomendado por la OMS, que es de $85 \%$. Se discuten las implicaciones que tiene, para el Programa de Prevención y Control de la Tuberculosis, la tasa elevada de abandonos y la probabilidad de circulación de cepas de M. tuberculosis resistente. Se identificaron como necesidades urgentes: diseñar estrategias creativas para asegurar la adherencia al tratamiento, que consideren al paciente y no solamente a los servicios de salud; extender el uso de cultivos para conocer la farmacorresistencia, y evaluar la conveniencia de modificar los esquemas de retratamiento. La revisión de las tarjetas de control de tratamiento es una herramienta útil para la evaluación del programa.

Palabras clave: tuberculosis/terapia, resultado del tratamiento; México ure or relapse. Conclusions. Proportion of cure rate $(58 \%)$ compares unfavorably with W HO recommendations (85\%). Implications of a high dropout rate and probability of circulation of resistant strains of $\mathrm{M}$ tuberculosis are discussed. Creative strategies to reinforce patient compliance which take into account the patient and not only the health services, extension of cultures to known M. tuberculosis drug resistance and evaluation of modifications to drug regimens are proposed. Review of treatment control cards is a useful tool for program evaluation.

Key words: tuberculosis/therapy, treatment outcome; Mexico
$\mathrm{E}$ n México la tuberculosis continúa siendo endémica; ${ }^{1}$ de acuerdo con los informes del Programa de Prevención y Control de la Tuberculosis, las tasas de incidencia han aumentado durante los últimos 10 años, de 14.4 casos por 100000 habitantes en 1986 a 18.2 casos por 100000 habitantes en $1996 .{ }^{*}$ Sin embargo, es probable que el número real de casos sea mucho más alto; se ha estimado que la tasa de morbilidad anual es de 50 casos por 100000 habitantes y que en la última década se han presentado por lo menos 27000 casos adicionales. ${ }^{2}$ En el estado de Morelos, destaca en particular el incremento en la tendencia de casos durante los últimos años, ya que ha pasado de 17 casos por 100000 habitantes en 1990 a 19.4 casos por 100000 habitantes en 1996. En esa entidad, al igual que en el resto del país, se siguen los lineamientos señalados por la Norma Oficial Mexicana para la Prevención y Control de la Tuberculosis en la Atención Primaria a la Salud, que se basa en la vacunación

\footnotetext{
* Coordinación de Vigilancia Epidemiológica, Secretaría de Salud. Programa de Control y Prevención de la Tuberculosis. Programa Sustantivo, 1997. Documento no publicado.

₹ Servicios de Salud en Morelos. Programa Estatal de Prevención y Control de la Tuberculosis, 1996. Documento no publicado.
}

con BCG al nacimiento, el diagnóstico mediante baciloscopías y el tratamiento supervisado con fármacos de presentación integrada. ${ }^{3}$ El objetivo del presente estudio fue analizar de manera retrospectiva la eficacia y la eficiencia del tratamiento antituberculoso administrado por la Secretaría de Salud (SSA) entre 1992 y 1996 en las jurisdicciones sanitarias de Cuernavaca y Cuautla, mediante la revisión de las tarjetas de control de tratamiento de pacientes con tuberculosis. Se hizo el análisis de las cohortes de pacientes tuberculosos que anualmente recibieron tratamiento en las unidades de salud, estrategia de evaluación recomendada por la Organización Mundial de la Salud (OMS). ${ }^{4}$

\section{Material y métodos}

El estado de Morelos comprende tres jurisdicciones sanitarias: Cuernavaca, Cuautla y Zacatepec. Se estudiaron los casos de tuberculosis que atendió la SSA en las jurisdicciones sanitarias de Cuernavaca y Cuautla; la de Cuernavaca está integrada por 11 municipios, con una población total de 590931 habitantes; la jurisdicción de Cuautla está integrada por 16 municipios, con una población total de 404038 habitantes. Entre ambas representan $80 \%$ de la población estatal. La jurisdicción de Cuernavaca es predominantemente ur- 
bana; la mayoría de la población económicamente activa está dedicada a la industria manufacturera y prestadora de servicios. En cambio, la jurisdicción de Cuautla tiene mayor proporción de áreas rurales, de tal modo que cerca de una tercera parte de la población económicamente activa se dedica a la agricultura. Si bien se ha incrementado el acceso a los servicios durante los últimos años, en Cuautla $8.5 \%$ de la población es analfabeta; $3.17 \%$ de las viviendas no tiene agua entubada y $4.9 \%$ tiene piso de tierra. En el caso de Cuernavaca, esos indicadores son más altos, aunque persisten las deficiencias: existe $6 \%$ de analfabetismo, $1.6 \%$ de viviendas sin agua entubada y $3 \%$ con piso de tierra. $^{5}$

Los servicios de salud que componen la infraestructura de la SSA comprenden 149 unidades de atención primaria (centros de salud, unidades auxiliares de salud, casas de salud), de las cuales 72 se ubican en la jurisdicción de Cuernavaca y 77 en la de Cuautla. Existen cuatro hospitales generales -dos en cada jurisdicción- y seis laboratorios -tres en cada jurisdicción- que realizan estudios de micobacteriología; exclusivamente se realizan frotis de baciloscopía con la técnica de Ziehl-Neelsen. No se realizan cultivos ni estudios de drogosusceptibilidad de Micobacterium tuberculosis.

El Programa Estatal de Prevención y Control de la Tuberculosis* proporcionó datos acerca de la incidencia de casos de tuberculosis para las jurisdicciones sanitarias de Cuernavaca y Cuautla.

Se revisaron las tarjetas de control de tratamiento antituberculoso -disponibles en las unidades de atención primaria y en los hospitales de la SSA- pertenecientes a pacientes que recibieron atención entre 1992 y 1996 en las jurisdicciones sanitarias de Cuernavaca y Cuautla. Alumnos de la Maestría en Salud Pública y de la Especialización en Enfermería en Salud Pública del Instituto Nacional de Salud Pública recolectaron, mediante un formato estandarizado, la información pertinente.

Se utilizaron las definiciones recomendadas por la Norma Oficial Mexicana para la Prevención y Control de la Tuberculosis en la Atención Primaria a la Salud:

Caso nuevo: el enfermo en quien se establece por primera vez el diagnóstico de tuberculosis y que se notifica. Retratamiento: el que es administrado por el médico especialista ante un caso de tuberculosis multitratado o cuando fracasa el tratamiento de corta duración.

\footnotetext{
* Servicios de Salud en Morelos. Programa Estatal de Prevención y Control de la Tuberculosis, 1996. Documento no publicado.
}

Tratamiento supervisado: el que se proporciona en los establecimientos de salud bajo la vigilancia del personal que presta el servicio, para garantizar la toma de dosis del medicamento por parte del enfermo tuberculoso.

Cura bacteriológica: cuando el paciente completa su tratamiento con la desaparición de los síntomas y signos clínicos, y se realizan dos o más baciloscopías o cultivos con resultados negativos consecutivos al término del tratamiento.

Cura probable: cuando el paciente completa su tratamiento con la desaparición de los síntomas y signos clínicos, sin comprobación bacteriológica.

Abandono: cuando el paciente deja de asistir a la clínica por más de 15 días después de su última cita.

Fracaso: persistencia de baciloscopías o cultivos positivos después del quinto mes de tratamiento.

Muerte: cuando el paciente fallece durante el tratamiento. Recaída: la reaparición de bacilos en la expectoración o en otros especímenes, después de haber egresado del tratamiento por curación.

Adicionalmente, respecto al nivel socioeconómico se estratificó a los pacientes de acuerdo con la Clasificación Mexicana de las Ocupaciones del Instituto Nacional de Estadística, Geografía e Informática (INEGI): en el nivel alto se ubicó a los profesionistas, gerentes y propietarios de comercios; en el nivel medio, a los empleados, oficinistas, amas de casa y estudiantes, y en el nivel bajo, a los obreros, campesinos, desempleados y reclusos.

Los formatos de recolección fueron revisados y validados por un solo investigador. La información sociodemográfica se analizó considerando al total de pacientes. Los datos sociodemográficos se compararon con los correspondientes a la información proporcionada por el INEGI para las áreas de estudio. ${ }^{7}$ Los resultados de los esquemas de tratamiento se analizaron según si correspondían al primer tratamiento del paciente (caso nuevo) o al segundo o subsecuente (retratamiento). Se evaluaron la eficacia (proporción de pacientes curados respecto al total de pacientes que terminaron tratamiento) y la eficiencia del tratamiento (proporción de pacientes curados respecto al total de pacientes que iniciaron tratamiento).

Se compararon las variables categóricas mediante la prueba $\chi^{2}$, y las variables continuas, mediante la prueba de $t$. Se calcularon razón de momios (RM) e intervalos de confianza (IC) al 95\%, asociados al abandono del tratamiento. Se utilizó el análisis de regresión logística no condicionado para determinar los factores asociados de manera independiente con el abandono del tratamiento. Las variables que se utilizaron en 
los modelos se seleccionaron de acuerdo con su significancia estadística en el análisis univariado y con su relevancia biológica. Se utilizaron los programas SAS y Epi Info.

\section{Resultados}

La información se recopiló entre octubre y diciembre de 1996. Se visitaron las 149 unidades de atención primaria y los cuatro hospitales de la SSA ubicados en las jurisdicciones sanitarias de Cuernavaca y Cuautla. Se encontraron las tarjetas de 288 pacientes, 260 de los cuales correspondieron a casos nuevos; en conjunto esos pacientes recibieron 311 tratamientos, $242(85 \%)$ de los cuales correspondieron a tratamientos supervisados.

En el cuadro I se muestra el número de pacientes notificados por año en cada jurisdicción, así como el número de pacientes de quienes se encontró tarjeta de tratamiento; no se incluyeron cuatro pacientes para los cuales no se consignó la fecha de tratamiento. La proporción de tarjetas estudiadas respecto al total de pacientes tuberculosos notificados por la SSA en ambas jurisdicciones en los años 1992 a 1995 fue de 60\%. No se comparó el número de tarjetas para 1996 por no contar con el dato sobre casos notificados. Se encontró una mayor proporción de tarjetas en la jurisdicción de Cuernavaca (72\%) que en la de Cuautla (49\%).

Las características sociodemográficas de los 288 pacientes en comparación con la población general establecida en el área, de acuerdo con el INEGI, ${ }^{5}$ se muestran en el cuadro II. La población de pacientes tuberculosos fue de mayor edad, comparada con la media poblacional, y pertenecía a niveles socioeconómicos más bajos que el de la población general.
Desde el punto de vista clínico en $80 \%$ de los pacientes se obtuvo diagnóstico bacteriológico mediante baciloscopía. De los 288 pacientes 40 (14\%) presentaron localizaciones extrapulmonares: linfáticas $(n=18)$, genitourinaria $(n=10)$ y otras $(n=12)$. Cincuenta y cuatro pacientes $(19 \%)$ presentaron enfermedades asociadas: la mayoría correspondió a diabetes $(n=36)$, alcoholismo $(n=18)$ y neoplasias $(n=2)$; solamente un paciente presentó VIH/SIDA y tres presentaron combinaciones.

En el cuadro III se muestran los resultados del tratamiento según si se trató de casos nuevos o de retratamiento. Se analizaron 246 esquemas de tratamiento y no se consideraron en este análisis 51 esquemas de tratamiento que no se habían concluido, 10 que correspondieron a pacientes que fueron transferidos a otra unidad y cuatro de los que se desconocía si correspondieron a caso nuevo o a retratamiento. De este modo, $58 \%$ de los pacientes presentaron curación bacteriológica o probable. La frecuencia de curación fue mayor en los casos nuevos $(61 \%)$ que en los retratamientos $(38 \%)(p<0.05)$. Un número considerable de pacientes abandonó el tratamiento $(18 \%)$. La frecuencia de abandonos fue mayor en los pacientes retratados (32\%) que en los casos nuevos $(16 \%)(p<0.05)$; por lo tanto, la eficacia de los tratamientos fue de $71 \%$, y resultó mayor en los casos nuevos $(73 \%)$ que en los retratamientos (57\%), aunque la diferencia no fue estadísticamente significativa. La eficiencia, por su parte, fue de $58 \%$ y mayor en los casos nuevos $(61 \%)$ que en los retratamientos $(38 \%)(p<0.01)$.

Se analizó la asociación de variables sociodemográficas (edad, sexo, nivel socioeconómico), lugar de atención (Cuernavaca o Cuautla), localización de la tuberculosis, enfermedad asociada, año de diagnóstico,

\section{Cuadro I \\ Pacientes tuberculosos notificados por la SSA en Cuernavaca y Cuautla, Morelos. México, 1992-1996}

\begin{tabular}{|c|c|c|c|c|c|c|c|}
\hline \multirow[b]{2}{*}{ Año } & \multicolumn{3}{|c|}{ Cuernavaca } & \multicolumn{3}{|c|}{ Cuautla } & \multirow[b]{2}{*}{ Total de tarjetas } \\
\hline & Casos notificados* & Tarjetas & $\%$ & Casos notificados* & Tarjetas & $\%$ & \\
\hline 1992 & 45 & 29 & 64 & 48 & 24 & 50 & 53 \\
\hline 1993 & 38 & 28 & 73 & 47 & 17 & 36 & 45 \\
\hline 1994 & 29 & 25 & 86 & 45 & 24 & 53 & 49 \\
\hline$\overline{1995}$ & 62 & 44 & 71 & 41 & 24 & 58 & 68 \\
\hline 1996 & ND & 42 & - & ND & 27 & - & 69 \\
\hline$\overline{T o t a l}$ & 174 & 168 & - & 181 & 116 & - & 284 \\
\hline
\end{tabular}

ND: no disponible

N ota: en cuatro pacientes no se consignó la fecha de inicio de tratamiento

*Fuente: Programa Estatal de Prevención y Control de la Tuberculosis, jurisdicciones sanitarias de Cuernavaca y Cuautla, Morelos 


\section{Cuadro II \\ Características sociodemográficas DE LOS PACIENTES TUBERCULOSOS ATENDIDOS por la SSA en Cuernavaca y Cuautla, Morelos. Méxıco, 1992-1996}

$\begin{array}{lccc}\text { Variable } & \begin{array}{c}\text { Población de estudio } \\ n=288\end{array} & \begin{array}{c}\text { Censo (1995) } \\ n=991498\end{array} & p \\ \begin{array}{l}\text { Edad } \\ \quad \text { Media (DE) }\end{array} & 39.3(21.05) & 24.1(18.2) & <.01 \\ \text { Sexo } & \text { No. (\%) } & \text { N o. (\%) } & \\ \quad \text { Masculino } & 159(55) & 485574(48.8) & \text { N S }\end{array}$

$\mathrm{N}$ ivel socioeconómico

\begin{tabular}{lrcc} 
Alto & 0 & $19594(6.8)$ & \\
\hline Medio & $157(54)$ & $122030(42.14)$ & $<.01$ \\
\hline Bajo & $71(25)$ & $147902(51.1)$ & \\
Cuernavaca & $170(59)$ & $588319(59.3) \quad$ N S \\
Cuautla & $118(41)$ & $403179(40.7)$ &
\end{tabular}

DE: desviación estándar

NS: no significativa

Fuente: referencia 5

tipo de tratamiento (autoadministrado o supervisado), número de tratamientos y antecedente de vacunación con BCG, con el abandono del tratamiento. Se encontró asociación estadísticamente significativa exclusivamente entre el abandono del tratamiento y el hecho de recibir dos o más tratamientos o pertenecer a un nivel socioeconómico bajo. La probabilidad de abandonar el tratamiento fue triple (RM 3.3, IC95\% 1.3-8.5, $p=0.01$ ) en los sujetos que recibían dos o más tratamientos en comparación con los que recibieron un solo esquema, y resultó doble (RM 2.2, IC95\% 1.0-4.9, p= 0.04) en aquellos pertenecientes al nivel socioeconómico bajo en comparación con los sujetos del estrato socioeconómico medio o alto (cuadro IV).

Se analizaron las causas de retratamiento en 34 esquemas. Se encontró que 11 pacientes iniciaron un nuevo esquema por haber abandonado el anterior; nueve por haber recaído después de un tratamiento previo, y dos por haber fracasado en un tratamiento anterior. Desafortunadamente en 11 esquemas se desconoció cuál fue la condición del paciente al iniciar un retratamiento.

\section{Discusión}

La tuberculosis es un problema de salud pública, ya que constituye la primera causa de muerte debida a un solo agente infeccioso; además, se ha considerado como un problema reemergente, de tal manera que su control se ha contemplado en el Programa de Reforma del Sector Salud 1995-2000. ${ }^{6}$ En particular, en el estado de Morelos se ha observado una tendencia al incremento en las tasas de morbilidad por tuberculosis en los últimos años, ${ }^{*}$ al igual que en el resto del

* Servicios de Salud en Morelos. Programa Estatal de Prevención y Control de la Tuberculosis, 1996. Documento no publicado.

\section{Cuadro III}

Resultado del tratamiento* en PaCientes tuberculosos atendidos por la SSA en Cuernavaca y Cuautla, Morelos. México 1992-1996

\begin{tabular}{|c|c|c|c|c|c|c|c|}
\hline \multirow[t]{2}{*}{ Resultados del tratamiento } & \multicolumn{2}{|c|}{ Todos los casos } & \multicolumn{2}{|c|}{ Casos nuevos } & \multicolumn{2}{|c|}{ Retratamientos } & \multirow[b]{2}{*}{$\mathrm{p}^{\ddagger}$} \\
\hline & No. & $\%$ & No. & $\%$ & $\overline{\mathrm{No}}$ & $\%$ & \\
\hline Curación bacteriológica & 78 & 32 & 72 & 34 & 6 & 18 & NS \\
\hline Curación probable & 65 & 26 & 58 & 27 & 7 & 20 & NS \\
\hline Subtotal curación & 143 & 58 & 130 & 61 & 13 & 38 & 0.01 \\
\hline Abandono & 45 & 18 & 34 & 16 & 11 & 32 & 0.02 \\
\hline Fracaso & 2 & 1 & 1 & 0.5 & 1 & 3 & NS \\
\hline Muerte & 7 & 3 & 6 & 3 & 1 & 3 & NS \\
\hline Desconocido & 49 & 20 & 41 & 19 & 8 & 23 & NS \\
\hline
\end{tabular}

* Se excluyen 51 esquemas de tratamiento no concluidos, 10 pacientes que fueron transferidos a otra unidad y cuatro de los que se desconoció si se trató de caso inicial o retratamiento

₹ Prueba $\chi 2$

NS: no significativa 


\section{Cuadro IV \\ Factores asociados al abandono del tratamiento EN PACIENTES TUBERCULOSOS ATENDIDOS POR la SSA en Cuernavaca y Cuautla, Morelos. MÉxico 1992-1996}

\begin{tabular}{lcccccccc} 
& \multicolumn{2}{c}{ Abandono } & & \multicolumn{2}{c}{ No abandono } & RM ajustada & $p$ \\
\cline { 6 - 8 } & No./total & $\%$ & & No./total & $\%$ & & \\
Tratamientos & & & & & & & \\
subsecuentes & $13 / 46$ & 28 & $22 / 214$ & 10 & 3.3 & 0.01 \\
\hline
\end{tabular}

Nivel socio-

\begin{tabular}{ccccccc} 
económico bajo & $19 / 40$ & 47 & $49 / 167$ & 29 & 2.2 & 0.04 \\
\hline & & $(1.0-4.9)$ &
\end{tabular}

país $^{1}$ (figura 1). Este estado se encuentra por arriba del promedio de casos notificados en el país durante 1996 (19.4 casos por 100000 habitantes vs 18.2 casos por 100000 habitantes). Lo anterior ocurre a pesar de que en esa entidad, al igual que en el resto del país, se siguen los lineamientos señalados por el Programa de Prevención y Control de la Tuberculosis a través de la norma oficial correspondiente.

El análisis de las cohortes de pacientes tuberculosos mediante la revisión de tarjetas de control de tratamiento constituye una herramienta útil para evaluar la eficacia y la eficiencia del tratamiento antituberculoso en una región, y es la recomendada por la OMS. ${ }^{4}$ A partir de la revisión de tarjetas se observó que la tasa de curación en el área de estudio está por debajo de lo recomendado por la OMS, la cual establece que el mínimo aceptable de pacientes curados debe ser de $85 \%{ }^{7}$ La frecuencia de curación fue de $58 \%$ en los paciente que iniciaron tratamiento (eficiencia) y de $71 \%$ en los pacientes que terminaron tratamiento (eficacia). Dos circunstancias explican esta baja tasa de curación: en primer lugar, la baja eficiencia se explica por la frecuencia tan alta de abandonos del tratamiento $(18 \%)$, la cual fue significativamente mayor en pacientes que recibieron retratamientos (32\%) que en los casos nuevos $(16 \%)$. Al analizar la eficacia del tratamiento se encontró que, aun excluyendo a los pacientes que abandonaron el tratamiento, la tasa de curación continúa siendo baja $(71 \%)$, lo cual se explica por la frecuencia de recaídas, fracasos y defunciones. Adicionalmente se observó que por lo menos una tercera parte de los tratamientos subsecuentes se iniciaron después de recaídas o fracasos del tratamiento anterior. Estos datos indican la posibilidad de que exista trans-

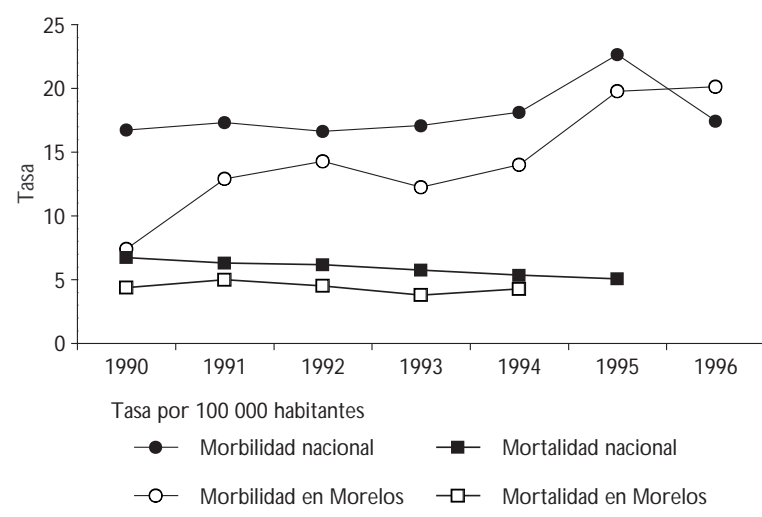

FIGURA 1. MORTALIDAD Y MORBILIDAD POR TUBERCULOSIS PULMONAR Y EXTRAPULMONAR EN EL ESTAdO DE Morelos. MÉxico, 1990-1996

misión de $M$. tuberculosis resistente a los antimicrobianos de primera línea.

Es importante señalar que la técnica de revisión de tarjetas de tratamiento resulta un método eficiente y poco costoso para evaluar la eficacia y la eficiencia del tratamiento. Si bien no es equivalente al diseño prospectivo de un ensayo clínico, el cual permitiría controlar todas las variables que afectan los resultados de una intervención, la mayor aportación que proporciona este tipo de análisis es la posibilidad de evaluar la eficacia y la eficiencia de los programas en las condiciones en las que se llevan a cabo en las unidades de salud. Por otro lado, por su carácter retrospectivo, tiene limitaciones importantes: la principal reside en no haber podido evaluar a la totalidad de pacientes que fueron tratados por haberles diagnosticado tuberculosis en el periodo de estudio, puesto que sólo se localizaron las tarjetas de tratamiento de $60 \%$ de los casos notificados durante ese lapso. Adicionalmente en $16 \%$ de las tarjetas fue imposible evaluar el resultado porque el dato no fue registrado en las mismas, lo cual significa que se evaluó aproximadamente $50 \%$ de los casos notificados por la SSA en ese periodo. Las tarjetas perdidas y aquellas en las que no se registró el resultado del tratamiento pudieron haber correspondido a casos problemáticos. Por lo tanto, es posible que la frecuencia de abandonos, fracasos y muertes sea superior a lo aquí descrito. Por otro lado, la pérdida de tarjetas y su llenado incompleto reflejan problemas administrativos en el seguimiento de los pacientes.

Se han descrito múltiples factores que influyen sobre la adherencia al tratamiento, entre los que se in- 
cluyen las características del sistema de salud, ${ }^{8}$ el tipo de esquema de administración de medicamentos que se aplica, ${ }^{9}$ las características del paciente ${ }^{10}$ y lo adecuado de la relación médico-paciente. ${ }^{11}$ La supervisión directa del tratamiento ha sido uno de los factores determinantes para lograr la adherencia al tratamiento. ${ }^{12-14} \mathrm{El}$ programa de control de la tuberculosis en el área de estudio se realiza de acuerdo con lo indicado por la norma oficial y considera varios de los factores anteriormente señalados: el tratamiento es supervisado por personal de salud en las instituciones de salud; se utilizan medicamentos orales en presentación consolidada, y la frecuencia de administración es diaria durante dos meses y, posteriormente, tres veces por semana durante cuatro meses, acciones que se han identificado como fundamentales para mejorar la adherencia. En esas condiciones se encontró que el nivel socioeconómico bajo se asoció a una menor adherencia, lo cual contrasta con los estudios internacionales recientes al respecto, pues la mayoría no ha detectado características específicas de los pacientes que se encuentran en riesgo de abandonar el tratamiento. ${ }^{15} \mathrm{El}$ hecho de provenir de estratos socioeconómicos desprotegidos condiciona el estilo de vida, las creencias en relación con la salud y el acceso a los servicios de salud, factores que se deben considerar con el objeto de mejorar la adherencia al tratamiento en este grupo. ${ }^{16}$

El programa de control requiere diseñar estrategias creativas que mejoren la adherencia al tratamiento en los pacientes de estratos socioeconómicos más bajos, puesto que el seguimiento estricto a la norma no está siendo suficiente. De acuerdo con la Norma Oficial para la Prevención y Control de la Tuberculosis en la Atención Primaria a la Salud, ${ }^{3}$ se recomienda que el tratamiento se administre en las instituciones de salud y que sea el personal de salud quien supervise que el paciente ingiera el medicamento. Sin embargo, este requerimiento no considera factores como el costo que ello significa en transporte y la pérdida de días de trabajo; en realidad, resulta más cómodo para el personal de salud que para el paciente mismo.

Recientemente se ha instaurado en algunas zonas del país el Programa de Tratamiento Antituberculoso Estrictamente Supervisado que permite que el paciente reciba el tratamiento antituberculoso en diferentes instituciones de salud de acuerdo con su conveniencia y no con la que se le asigna por razones burocráticoadministrativas. Los resultados preliminares comienzan a mostrar que esta estrategia está mejorando las tasas de curación. ${ }^{17}$ En otros países se han puesto en práctica estrategias creativas como son la administración del tratamiento en la comunidad,,$^{18-20}$ en sitios tales como hogares, centros de trabajo y lugares de re- creación como los bares, ${ }^{21}$ con el apoyo de personas de la comunidad..$^{22}$ Es necesario que las modificaciones al programa estén orientadas al paciente y no a los sistemas de salud..$^{23}$ A partir de los resultados que se plantean aquí, se recomienda flexibilizar los criterios de supervisión del tratamiento y evaluar el tratamiento administrado en la comunidad y con el apoyo de sus mismos miembros.

El abandono del tratamiento tiene implicaciones graves, como lo han demostrado diversos estudios: es uno de los factores más importantes que condicionan la aparición de cepas resistentes ${ }^{24,25}$ y es un factor que determina la transmisión prolongada de $M$. $t u$ berculosis. ${ }^{26}$

Si bien se carece de información sobre la frecuencia de resistencia a fármacos en el área, los datos sobre las causas de reingreso a tratamiento pueden orientar en ese sentido: de 34 esquemas de retratamiento, 11 se debieron a que el paciente recayó o fracasó en un tratamiento anterior. Esto puede deberse a que la resistencia antimicrobiana a $M$. tuberculosis está ocasionando fallas en el tratamiento de los pacientes.

Los datos disponibles sobre drogosusceptibilidad de $M$. tuberculosis en el país son los proporcionados por el Departamento de Micobacterias del Instituto Nacional de Diagnóstico y Referencia Epidemiológicos (INDRE). En 1811 cepas analizadas entre 1989 y 1993 se encontró que la frecuencia de resistencia inicial era de 4.2 a $9 \%$, y de resistencia adquirida, de 59.4 a $74.7 \%$. $^{2}$ En población hospitalaria, Sifuentes encontró resistencia antimicrobiana a isoniacida en $19 \%$ y a rifampicina en $12 \%$ en 84 aislados clínicos, ${ }^{27}$ y Olvera notificó resistencia de $62 \%$ a isoniacida y $61 \%$ a rifampicina en 232 pacientes retratados. ${ }^{28}$ Estos datos señalan la importancia que puede tener la resistencia de $M$. tuberculosis en México y hacen evidente la necesidad de practicar estudios de drogosusceptiblidad.

La tasa de curación por debajo de lo recomendado en los tratamientos concluidos y las recaídas posteriores al tratamiento que se detectaron al revisar las tarjetas indican que es probable que esos pacientes sean portadores de cepas resistentes a los antimicrobianos utilizados en los esquemas primarios y que estén ocasionando la transmisión prolongada de la infección y la aparición de casos nuevos con resistencia a antimicrobianos. La administración del tratamiento supervisado disminuye la frecuencia de resistencia, ${ }^{29}$ y es la estrategia más importante que debe ponerse en práctica en el corto plazo para mejorar el programa de prevención y control, y garantizar así la curación de los casos nuevos de tuberculosis. Adicionalmente, para poder controlar este problema es importante conocer la frecuencia de resistencia antimicrobiana tanto 
en casos nuevos de tuberculosis como en pacientes que han recibido tratamientos previamente. Se ha identificado este problema en todo el país y actualmente la SSA, la OMS y los Centros para la Prevención y el Control de Enfermedades de Estados Unidos de América conducen, de manera conjunta, un estudio para investigar la farmacorresistencia de $M$. tuberculosis en aislados clínicos provenientes de casos nuevos de tuberculosis. *

De acuerdo con la norma oficial ${ }^{3}$ se recomienda realizar cultivo y estudio de drogosusceptiblidad solamente en los casos retratados y en los pacientes con tuberculosis extrapulmonar; en casos nuevos se realiza exclusivamente cuando, ante la presencia de sintomatología, se obtienen por lo menos seis baciloscopías negativas y con fines de monitoreo de resistencia primaria. En el área que se estudió se encontró que no existe la infraestructura necesaria para la realización de cultivos y que tampoco se envían muestras al Laboratorio de Referencia Nacional en el INDRE. Con base en la frecuencia de fracasos y recaídas se considera pertinente valorar la necesidad de realizar cultivos en una mayor proporción de casos nuevos para conocer la frecuencia de resistencia inicial y reforzar la realización de cultivos en los casos retratados.

Para llevar a cabo todo esto habrá que reforzar la infraestructura de laboratorio existente en el área, así como los mecanismos administrativos que aseguren la referencia. Los resultados permitirán conocer la necesidad de disponer de medicamentos de segunda línea que puedan ser administrados oportunamente. Se descubrió que por lo menos 11 pacientes recayeron a pesar de haber recibido el tratamiento reforzado que recomienda la norma oficial (isoniacida, rifampicina, piracinamida y etambutol). Ante la frecuencia de recaídas y fracasos repetidos debe considerarse la conveniencia de utilizar cinco fármacos como recomienda la OMS, ${ }^{30}$ puesto que es probable que se trate de cepas resistentes a por lo menos tres de los fármacos que se están administrando.

La evaluación de la eficacia y la eficiencia del tratamiento mediante la revisión de las tarjetas de control proporciona información útil a los responsables de los programas; en particular aquí se documentaron tasas altas de tuberculosis a pesar de que en el área se siguen los lineamientos señalados en la norma oficial. Los datos de este estudio indican que el abandono del tratamiento continúa siendo importante a pesar de que el mismo se lleva a cabo en condiciones supervisadas, y que los sujetos con mayor probabilidad de abando-

\footnotetext{
* Balandrano S., comunicación personal.
}

nar los tratamientos son los provenientes de estratos socioeconómicos bajos y aquellos que reciben tratamientos subsecuentes. Adicionalmente se encontró que, incluso en aquellos pacientes que terminan tratamiento, se obtiene una tasa de curación inferior a la esperada, y que al menos una tercera parte de los retratamientos se inician por fracasos o por recaídas posteriores al tratamiento, lo cual indica la probabilidad de circulación de cepas resistentes a los antimicrobianos. Los datos obtenidos de esta manera permiten identificar los problemas que surgen al aplicar el programa en los centros de atención primaria y en los hospitales. Las alternativas de solución que se proponen aquí implican un enfoque múltiple que requiere del diseño de estrategias creativas que involucren tanto el diagnóstico de los casos como el tratamiento de los mismos.

\section{Agradecimientos}

Los autores agradecen la colaboración de las autoridades de la Secretaría de Bienestar Social del Estado de Morelos, en particular a los doctores Carlos Javier Martínez de León, Salvador Casares Queralt, Concepción Tavera Díaz, Manuel de Jesús Padilla Pimentel, Gloria del Carmen Pérez Ochoa y Héctor Romero Zúñiga, así como a la enfermera Alma Martínez y al personal de las unidades de salud de la SSA de las jurisdicciones sanitarias de Cuernavaca y Cuautla, por su apoyo para la obtención de la información; se agradece también a los alumnos de la Maestría en Salud Pública (generación 1996-1997), los doctores Ramón Dorantes, Maricela Godínez, Augusto Hernández, César Hernández, Arturo Méndez, Enrique Navarro, Ramón Valenzuela, a los licenciados en enfermería Luz Rosario Martínez y Rey Arturo Salcedo Alvarez, y a las alumnas de la Especialidad en Enfermería en Salud Pública (generación 1996-1997), enfermeras Adaena Amaro y Juana Hernández Soto, por su apoyo en la recolección de la información y en el análisis de los datos.

\section{Referencias}

1.Tapia R, Ruiz C, Ferreira E. Epidemiología de la tuberculosis en México. En: Sada E, Sifuentes J, ed. Tuberculosis. México, D.F.: McG raw-Hill/Interamericana Editores, 1995:761-788 (Ramiro HM, Saita OK, ed. Temas de Medicina Interna, vol 3).

2. Valdespino JL, Velasco O, Escobar A, ed. Enfermedades tropicales en México. Diagnóstico, tratamiento y distribución geográfica. México, D.F.: Secretaría de Salud, 1994:215-225. 
3. Secretaría de Salud. N orma O ficial para el Control y Prevención de la Tuberculosis en la Atención Primaria a la Salud. N orma N O. N O M-006SSAA2-1993. Diario O ficial de la Federación, 1995 enero 26.

4. Chaulet $P$, Zidouni N . Evaluation of applied strategies of tuberculosis control in the developing world. En: Reichman L, Hershfield, ed. Tuberculosis. A comprehensive international approach. $N$ ueva York: Marcel Dekker, 1993:601-627.

5. Instituto N acional de Estadística, Geografía e Informática. Anuario Estadístico del Estado de Morelos. México, D.F.:Talleres Gráficos del Instituto N acional de Estadística, Geografía e Informática, 1995.

6. Secretaría de Salud. Programa de Reforma del Sector Salud 1995-2000. Diario 0 ficial de la Federación, 1996 marzo 11.

7. Stylbo K, Salamao A. National Tuberculosis Control Programs. En: Reichman L, Hershfield, ed. Tuberculosis. A comprehensive international approach. N ueva York: Marcel D ekker, 1993:573-600.

8. Farmer P, Robin S, Ramilus SL, Kim JY.Tuberculosis, poverty and "compliance": Lessons from rural Haiti. Semin Respir Infect 1991;6:254-260.

9. Kumaresan JA, Maganu ET. C ase holding in patients with tuberculosis in Botswana. BMJ 1992;305:340-341.

10. Ferrer $X$, Kirschbaum A, Toro J, Jadue J, Muñoz M, Espinoza A. A dherencia al tratamiento de la tuberculosis del adulto en Santiago, Chile. Bol O ficina Sanit Panam 1991; 111:423-431.

11. Pozsik CJ. Compliance with tuberculosis therapy. Med Clin N orth Am 1993;77:1289-1301.

12. Iseman MD, C ohn DL, Sbarbaro JA. Directly observed treatment of tuberculosis.We can't afford no to try it. N Engl J Med 1993;329:135-136. 13. Caminero JA, Pavon JM, Rodríguez-D e Castro F, Diaz F, Julia G, C ayla $J A$ et al. Evaluation of a directly observed six months fully intermittent treatment regimen for tuberculosis in patients suspected of poor compliance. Thorax 1996:51:130-133.

14. El-Sadr W, Medard F, Barthaud V. Directly observed therapy for tuberculosis: The Harlem Hospital experience, 1993. Am J Public Health 1996;86:1146-1149.

15. Sbarbaro J.The patient-physician relationship: Compliance revisited. Ann Allergy 1990;64:325.

16. Rubei AJ, Garro LC. Social and cultural factors in the successful control of tuberculosis. Public Health Rep 1992;107:626-636.
17.Yáñez-Velasco L, Q uiroz G, Rodríguez J. Use of dots in pilot areas of Mexico. Intern J Tub Lung D is 1996;1:S68.

18. Chaulk P, Moore-Rice K, Pizzo R, Chaisson R. Eleven years of community-based directly observed therapy for tuberculosis. JAMA 1995;274: 945-951.

19.W ilkinson D. High compliance tuberculosis treatment programme in a rural community. Lancet 1994;343:647-648.

20.Van der W erfTS, Dade GK,Van der MarkTW. Patient compliance with tuberculosis treatment in $\mathrm{G}$ hana: Factors influencing adherence to therapy in a rural service programme. Tubercle 1990;71:247-252.

21. Kline SE, Hedemark L, Davies S. O utbreak of tuberculosis among regular patrons of a neighborhood bar. N Engl J Med 1995;333:222-227.

22. W ilkinson D, Davies $G R$, Connolly C. Directly observed therapy for tuberculosis in rural South Africa, 1991 through 1994.Am J Public Health 1996; 86:1094-1097.

23. Pozsik CJ. Compliance with tuberculosis therapy. Med Clin N orth Am 1993;77:1289-1301.

24. Goble $M$, Iseman MD, Madsen LA, W aite D, Ackerson L, Horsburgh CR. Treatment of 171 patients with pulmonary tuberculosis resistant to isoniazid and rifampin. N Engl J Med 1993;399:527-532.

25. Mahmoudi A, Iseman MD. Pitfalls in the care of patients with tuberculosis. JAMA 1993;270:65-68.

26. G angadharam P. D rug resistance in tuberculosis. En: Reichman L, Hershfield, ed. Tuberculosis. A comprehensive international approach. N ueva York: Marcel Dekker, 1993:293-328.

27. Sifuentes-O sornio J, Ponce-De León LA, Camacho-Mezquita E, Bobadilla-del Valle M, Infante-Suárez ML, Ramírez-Fernández $\mathrm{N}$ et al. Resistencia de Mycobacterium tuberculosis en pacientes mexicanos. I.C aracterísticas clínicas y factores de riesgo. Rev Invest Clin 1995;47: 273-281.

28. O Ivera-C astillo R, Pérez-González LE. Resistencia secundaria en tuberculosis. Rev Inst Nal Enf Resp 1993;6:185-190.

29. Frieden TR, Fujiwara PI, W ashko RM, Hamburg MA. Tuberculosis in N ew York City, turning the tide. N ew Engl J Med 1995;333:229-233.

30. Sudre $P$, Ten-Dan $G, C$ han $C$, Kochi A. Tuberculosis in the present time: A global overview of the tuberculosis situation. Ginebra: World Health O rganization, D ocumento W HO/tub/91.158, 1991. 